\title{
In Vitro and In Vivo Accuracy Evaluation of Computer Guide Surgery for Dental Implant Placement Associated with Three Mini-implants Used as Precision Pins
}

Guarnieri Renzo $^{*}$, Ippoliti Stefano, Stefanelli Luigi Vito, Di Carlo Stefano, Pompa Giorgio

Department of Dental and Maxillofacial Science, School of Dentistry, University La Sapienza, Roma, Italy

\section{Abstract}

Objective: to evaluate in vitro and in vivo the accuracy and the precision of guided implant surgery, associated with three mini-implants placement used as precision pins.

Materials and Methods: For in vitro evaluation, two acrylic resin model with artificial gum were prepared to represent edentulous mandibles. For the next in vivo evaluation two patients were treated using the same method. Three mini-implants used as reference points were placed before diagnostic and therapeutic procedure. Pre-and post-implantation CBCT images were superimposed using digital processing image software to evaluate the linear and angular deviations between the virtual planning data and the surgical results.

Results: In vitro: The mean angular deviation between the virtual and actual positions of twelve placed implants was 2.4 degrees (SD 0.168). The mean depth deviation at the level of the implant shoulder (D1) was $0.23 \mathrm{~mm}$ (SD 0.021), the depth deviation at the implant center (D2) was $0.21 \mathrm{~mm}$ (SD 0.007), and the depth deviation at the level of apical point (D3) was $0.14 \mathrm{~mm}$ (SD 0.014). The mean shoulder mesial radial deviation (SMR) was $0.30 \mathrm{~mm}$ (SD 0.007), the mean shoulder distal deviation was $0.27 \mathrm{~mm}$ (SD 0.028), the mean implant center deviation was $0.21 \mathrm{~mm}$ (SD 0.17), and the mean apex radial (ARP) deviation was $0.15 \mathrm{~mm}$ (SD 0.021). and $0.27 \mathrm{~mm}$ (SD 0.19), respectively. The differences were found not statistically significant In vivo: The mean angular deviation between the virtual and actual positions of twelve placed implants was 3.0 degrees (SD 0.1167). The mean D1 deviation was $0.27 \mathrm{~mm}$ (SD 0.018), the mean D2 deviation was $0.29 \mathrm{~mm}$ (SD 0.041), and the mean D3 deviation was $0.17 \mathrm{~mm}$ (SD 0.019). The mean SMR deviation was $0.27 \mathrm{~mm}$ (SD 0.021); the mean SDR deviation was $0.34 \mathrm{~mm}(0.022)$ while the ARP mean deviation was $0.24 \mathrm{~mm}$ (SD0.248). The differences were found not statistically significant .

Conclusions: Results showed that the use of three mini-implants used as precision pin allows obtaining a promising accuracy in virtual implant placement.

\section{Introduction}

One of the most important developments in the field of implant dentistry is the introduction of computed tomography (CT) in conjunction with Computer-aided design/computer assisted manufacturing (CAD/CAM)-generated surgical guides. The visualization obtained from CT scans permits precise surgical planning for implant placement since anatomic limitations, bone morphology and the surgical site underneath the soft tissues can be evaluated precisely $[1,2]$. By using CT- assisted implant planning systems, it is possible to pre-surgically determine, with a high degree of accuracy and with $3 \mathrm{D}$ views, the best position and inclination for dental implant placement. To transfer the preoperatively planned implant position into the patient's mouth, surgical templates based on the preoperative set-up and virtual implant planning, are either fabricated manually in a dental laboratory or stereolithographically by CAD/CAM technology. Systems, which use this kind of procedure, are called "template-based" or "static". Other systems which use intraoperative optical tracking of the hand-piece position with cameras and guide the surgeon "real-time" are called "dynamic" [3]. Both static and dynamic systems have well-documented advantages and disadvantages, however, their application accuracy levels seem to be similar $[4,5]$. With the introduction of the cone-beam CT (CBCT) technology [6], which reduced radiation exposure [7], the operational availability of 3D diagnosis has extremely expanded in dental practices, and different static systems are now available for 3D guided implant treatment [3]. Several clinical studies have demonstrated the E-mail: renzoguarnieri@gmail.com original author and source are credited.

\section{Publication History:}

Received: August 05, 2016

Accepted: December 01, 2016

Published: December 032016

\section{Keywords:}

Mini-implants, Computer guide surgery, In vitro, In vivo, Computed tomography of dental implants [8-10]. However, different issues regarding this kind of technology are still open to debate. It requires a complex consequential protocol, which involved several steps: 1) the fabrication of a radiographic template, 2) the $\mathrm{CBCT}$ acquisition with the template in position, 3) the computer assisted implant planning, and 4) the fabrication and use of a surgical guide for drilling and implant insertion. Every step of the sequence is prone to errors [11]. Therefore, it is important to determine the most precise system for accurately and securely transferring the CAD plan to the surgical environment. The accuracy of various computer-guided implant treatment systems, defined as "the deviation in location or angle of the plan compared to the result" [11], has been well documented over the years [3,12,13]. Factors reported influencing the accuracy of the computer-guided approach in a negative way has been identified in bone supported guides, in the use of multiple templates, and in the lack of guide fixation [13].

"Corresponding Author: Prof. Renzo Guarnieri, Department of Dental and Maxillofacial Science, School of Dentistry, University La Sapienza, Roma, Italy;

Citation: Guarnieri R, Ippoliti S, Stefanelli LV, Di Carlo S, Pompa G (2016) In Vitro and In Vivo Accuracy Evaluation of Computer Guide Surgery for Dental Implant Placement Associated with Three Mini-implants Used as Precision Pins. Int J Surg Surgical Porced 1: 112. doi: http://dx.doi.org/10.15344/ijssp/2016/112

Copyright: (c) 2016 Guarnieri et al. This is an open-access article distributed under the terms of the Creative Commons Attribution License, which permits unrestricted use, distribution, and reproduction in any medium, provided the 
Citation: Guarnieri R, Ippoliti S, Stefanelli LV, Di Carlo S, Pompa G (2016) In Vitro and In Vivo Accuracy Evaluation of Computer Guide Surgery for Dental Implant Placement Associated with Three Mini-implants Used as Precision Pins. Int J Surg Surgical Porced 1: 112. doi: http://dx.doi.org/10.15344/ijssp/2016/112

Page 2 of 3

The hypothesis of the present study was that the placement of three mini-implants as reference points of guide fixation, might to overcome some of limitations related to these factors. Since mini-implants remain during the complete procedure as fixed reference points, the prosthetic guide can be inserted in a reliable and reproducible manner during the $\mathrm{CT}$ imaging as a future surgical template when screwed onto the mini-implants.

The accuracy of over mentioned technique, by evaluating the difference between planned and actual implant positions on pre- and post-operative CBCTs using a digital processing image software, was carried out.

\section{Materials and Methods}

Since the measuring the accuracy of implant placement vs. planning in vivo involves the acquisition of a post-surgical CBCT, which is medically unjustifiable in most clinical cases, and the use of human cadaver evolves ethical considerations, the first part of the present study was designed to assess the in vitro accuracy of the guided implant system associated with the use of three mini-implants placed as precision pins.

In vitro a clinical sequence of a computed guided implant treatment from planning to implant insertion, was conducted using acrylic resin mandibles covered with a silicone material to represent gingival tissue. Three mini-implants ( $7.5 \mathrm{~mm}$ long, $2.5 \mathrm{~mm}$ in diameter) were inserted covered with a silicone material to represent gingival tissue in every model in the retromolar area bilaterally and in the mandibular midline to established a tripodial distribution. Miniimplants remain during the complete procedure as fixed reference points. In this way, the prosthetic guide can be inserted in a reliable and reproducible manner during the $\mathrm{CT}$ imaging as a future surgical template when screwed onto the mini-implants. Tahmaseb et al $[14,15]$ previously proposed the use of reproducible fiducial markers, to obtain the placement of the implants in the correct vertical dimension. After insertion of the mini-implants, impressions were taken using impression coping and a polyether impression material. Models were poured with gypsum (WhipMix Quickstone Laboratory Stone, Louisville, KY, USA), using the mini-implant analogues. The diagnostic CT setup was delivered using an acrylic resin containing barium sulfate (Vivotac, Ivoclar Vivadent, Schaan, Liechtenstein). The CT template, which represents the future restoration, was then screwed onto the mini-implants before the CBCT recording, using a dedicated screw compex . Pre-operative CBCT scans of the models with the templates in place were acquired using Carestream CS 9300 (Carestream Health Italia s.r.l., Genova, Italy). Implant 3D software (Media Lab Ltd, La Spezia, Italy), was used to plan the virtual placement of the implants, and to realize the stereolithografic surgical guide in bio-compatible photopolymer material (MED610) by means of 3D printer (Stratasys Objet, Stratasys Corporate, Rehovot, Israel ), for the placement of six BioHorizons Tapered Internal implant ( $12 \mathrm{~mm} \times 4.6 \mathrm{~mm}$ ). After all implants had been placed according to Implant 3D surgical protocol, the jaws were again scanned with CBCT using the same image acquisition parameters and the same device. Following the double scanning protocol, the images were sent back to the manufacturer of the surgical guide to overlay the images. A GOM Inspect V.7.5 SR1 software program, (GOM Italia, Buccinasco (MI) Italy) was used to match pre- and post-operative implants positions. The following outcome variables were recorded:
1. Depth Deviation: The occluso-apical projection in millimetre of the postoperative implant axis on a plane through the preoperative implant axis. This was measured at the level of the implant shoulder (D1 depth deviation), at the implant center level [6 mm apically, (D2 depth deviation)], and at the level of apical point (D3 depth deviation).

2. Radial deviation - the projection in millimetre, of the postoperative implant axis on a plane perpendicular to the preoperative implant axis, measured at the level of the implant shoulder $(\mathrm{SMR}=$ shoulder mesial radial deviation, $\mathrm{SDR}=$ shoulder distal radial deviation) and at the level of apical point $(\mathrm{ARP}=$ apex radial deviation $)$.

3. Angular deviation (AD) - the angle (as part of $360^{\circ}$ ) between pre- and post-operative implant axes.

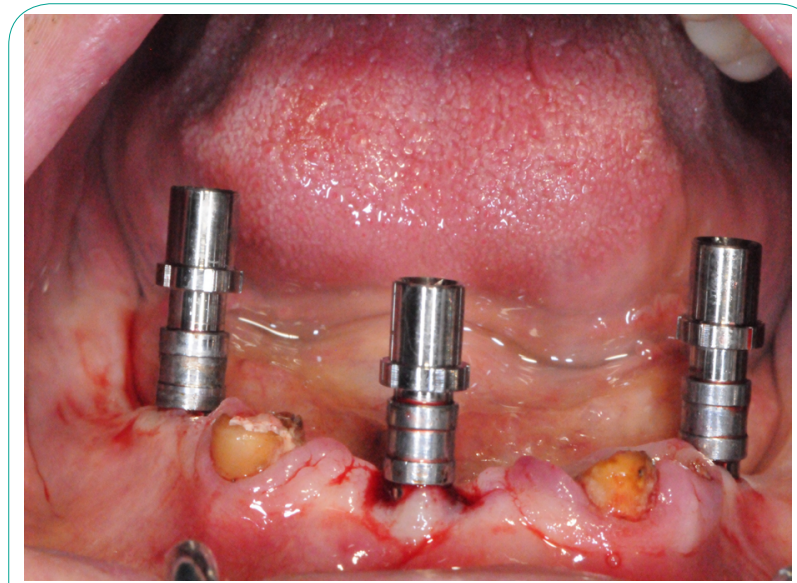

Figure 1: Three mini implants inserted in patient's jaw. Mini-implants remain during the complete procedure as fixed reference points. In this way, the prosthetic guide can be inserted in a reliable and reproducible manner during the CT imaging as a future surgical template when screwed onto the mini-implants.

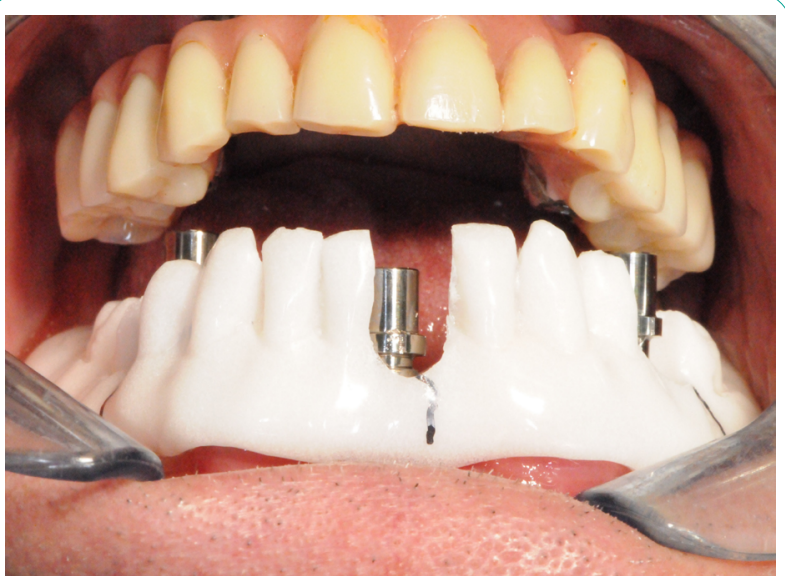

Figure 2: Surgical template designed using the same data as the planning software and fixed to 3 mini implants.

In the second part of the present study, having demonstrated in vitro the accuracy of the tested system, the clinical sequence of a computed guided implant treatment from planning to implant insertion, was performed on two patients, to assess in vivo outcomes. Patients underwent the same surgical protocol. An antimicrobial prophylaxis was administered with $500 \mathrm{mg}$ Amoxycillin twice daily for 5 days starting 1 hour before surgery. Local anesthesia was induced by infiltration with articaine/epinephrine and post-surgical analgesic treatment was performed with $100 \mathrm{mg}$ Nimesulid twice daily for 
Citation: Guarnieri R, Ippoliti S, Stefanelli LV, Di Carlo S, Pompa G (2016) In Vitro and In Vivo Accuracy Evaluation of Computer Guide Surgery for Dental Implant Placement Associated with Three Mini-implants Used as Precision Pins. Int J Surg Surgical Porced 1: 112. doi: http://dx.doi.org/10.15344/ijssp/2016/112

Page 3 of 3

3 days. Oral hygiene instructions were provided. Mucotomy was performed, bone drilled and BioHorizons Tapered Internal implant implants (BioHorizons, Birmingham, AL, USA) inserted as previously planned with CT-guided protocol. After all implants had been placed according to Implant 3D surgical protocol, the patient's jaws were again scanned with CBCT using the same image acquisition parameters and the same device. Following the double scanning protocol, the images were sent back to the manufacturer of the surgical guide to overlay the images. A GOM Inspect V.7.5 SR1 software program, (GOM Italia, Buccinasco (MI) Italy) was used to match pre- and post-operative implants positions.

\section{Data Analysis}

The $t$ test was used to compare the D1, D2, D3 depth deviation, the SRM, SRD, ARP radial deviation, and the angular deviation. Statistical significance was defined as $P<0.01$. Descriptive statistical analysis was performed with IBM SPSS Statistics version 19 (IBM Corp).

\section{Results}

In vitro test: Differences were observed between all measured distances (virtual versus actual). The D1 (at the level of the implant shoulder) distance had a mean value of $0.23 \mathrm{~mm}$ (SD 0.021). The D2 distance (at the level of implant center), and D3 distance (at the level of apical point) had mean values of $0.21 \mathrm{~mm}$ (SD 0.007) and $0.14 \mathrm{~mm}$ (SD 0.11), respectively. The mean apical point deviation was $0.15 \mathrm{~mm}$ (SD 0.29); while at the entry point the mean SMR and SDR radial deviations were $0.3 \mathrm{~mm}$ (SD 0.007), and $0.27 \mathrm{~mm}$ (SD 0.028), respectively. The mean angular deviation between the virtual and actual positions of twelve placed implants was 2.44 degrees (SD 1.98). The differences were found not statistically significant.

The maximum SMR error, the maximum SDR error, and the maximum horizontal apical error were $0.33 \mathrm{~mm}, 0.30 \mathrm{~mm}$, and 0.39 $\mathrm{mm}$, respectively. The maximum vertical error was $0.25 \mathrm{~mm}$, while the maximum angle error was 2.80 degrees. In table 1 are reported the in vitro results.

\begin{tabular}{|c|c|c|c|c|c|c|c|c|}
\hline \multicolumn{9}{|c|}{ Differences between planned and actual implant positions and angular deviations for each implant placed } \\
\hline Model & Implant no. & $\mathrm{D} 1(\mathrm{~mm})$ & $\mathrm{D} 2(\mathrm{~mm})$ & D3 (mm) & SMR & SDR & ARP & $\mathrm{AD}$ (degree) \\
\hline 1 & $\begin{array}{l}1 \\
2 \\
3 \\
4 \\
5 \\
6\end{array}$ & $\begin{array}{l}0.21 \\
0.24 \\
0.20 \\
0.23 \\
0.22 \\
0.25\end{array}$ & $\begin{array}{l}0.22 \\
0.24 \\
0.23 \\
0.20 \\
0.19 \\
0.21\end{array}$ & $\begin{array}{l}0.15 \\
0.16 \\
0.18 \\
0.11 \\
0.12 \\
0.14\end{array}$ & $\begin{array}{l}0.30 \\
0.31 \\
0.32 \\
0.33 \\
0.31 \\
0.33\end{array}$ & $\begin{array}{l}0.29 \\
0.25 \\
0.27 \\
0.26 \\
0.30 \\
0.27\end{array}$ & $\begin{array}{l}0.15 \\
0.18 \\
0.11 \\
0.17 \\
0.13 \\
0.15\end{array}$ & $\begin{array}{l}2.4 \\
2.3 \\
2.3 \\
2.4 \\
2.8 \\
2.5\end{array}$ \\
\hline 2 & $\begin{array}{l}1 \\
2 \\
3 \\
4 \\
5 \\
6\end{array}$ & $\begin{array}{l}0.24 \\
0.22 \\
0.23 \\
0.25 \\
0.23 \\
0.24\end{array}$ & $\begin{array}{l}0.23 \\
0.18 \\
0.24 \\
0.23 \\
0.21 \\
0.21 \\
\end{array}$ & $\begin{array}{l}0.15 \\
0.16 \\
0.13 \\
0.17 \\
0.13 \\
0.13\end{array}$ & $\begin{array}{l}0.27 \\
0.31 \\
0.30 \\
0.33 \\
0.28 \\
0.31 \\
\end{array}$ & $\begin{array}{l}0.25 \\
0.28 \\
0.29 \\
0.27 \\
0.29 \\
0.25\end{array}$ & $\begin{array}{l}0.13 \\
0.14 \\
0.16 \\
0.15 \\
0.11 \\
0.15\end{array}$ & $\begin{array}{l}2.5 \\
2.6 \\
2.4 \\
2.3 \\
2.1 \\
2.4\end{array}$ \\
\hline Mean & & 0.23 & 0.21 & 0.14 & 0.30 & 0.27 & 0.15 & 2.4 \\
\hline SD & & 0.021 & 0.007 & 0.014 & 0.007 & 0.028 & 0.021 & 0.168 \\
\hline$t$ & & 1.1438 & 0.1429 & 0.1306 & 1.6038 & 0.1562 & 0.6532 & 0.5798 \\
\hline $\mathrm{P}$ & & 0.2822 & 0.8892 & 0.8987 & 0.1340 & 0.8790 & 0.5284 & 0.5749 \\
\hline
\end{tabular}

Table 1: In vitro results.

D1 = depth deviation at the level of the implant shoulder; D2 = depth deviation at the implant center; D3 = depth deviation at the level of apical point; SMR $=$ shoulder mesial radial deviation; $\mathrm{SDR}=$ shoulder distal radial deviation; $\mathrm{ARP}=$ apex radial deviation at the level of apical point; $\mathrm{AD}=\mathrm{Angular}$ deviation

\begin{tabular}{|c|c|c|c|c|c|c|c|c|}
\hline Model & Implant no. & $\mathrm{D} 1(\mathrm{~mm})$ & $\mathrm{D} 2(\mathrm{~mm})$ & $\mathrm{D} 3(\mathrm{~mm})$ & SMR & SDR & ARP & $\mathrm{AD}$ (degree) \\
\hline 1 & $\begin{array}{l}1 \\
2 \\
3 \\
4 \\
5 \\
6\end{array}$ & $\begin{array}{l}0.28 \\
0.27 \\
0.24 \\
0.28 \\
0.27 \\
0.30 \\
\end{array}$ & $\begin{array}{l}0.32 \\
0.25 \\
0.28 \\
0.29 \\
0.27 \\
0.32 \\
\end{array}$ & \begin{tabular}{|l}
0.17 \\
0.20 \\
0.19 \\
0.18 \\
0.17 \\
0.16 \\
\end{tabular} & $\begin{array}{l}0.29 \\
0.26 \\
0.26 \\
0.28 \\
0.30 \\
0.27\end{array}$ & $\begin{array}{l}0.36 \\
0.33 \\
0.34 \\
0.35 \\
0.34 \\
0.36 \\
\end{array}$ & $\begin{array}{l}0.20 \\
0.25 \\
0.22 \\
0.23 \\
0.29 \\
0.25 \\
\end{array}$ & $\begin{array}{l}3.2 \\
3.0 \\
3.2 \\
3.8 \\
2.2 \\
2.9\end{array}$ \\
\hline 2 & $\begin{array}{l}1 \\
2 \\
3 \\
4 \\
5 \\
6\end{array}$ & $\begin{array}{l}0.26 \\
0.26 \\
0.28 \\
0.29 \\
0.26 \\
0.24 \\
\end{array}$ & $\begin{array}{l}0.28 \\
0.29 \\
0.31 \\
0.30 \\
0.29 \\
0.27 \\
\end{array}$ & \begin{tabular}{|l|}
0.15 \\
0.16 \\
0.13 \\
0.17 \\
0.18 \\
0.17 \\
\end{tabular} & $\begin{array}{l}0.24 \\
0.27 \\
0.29 \\
0.31 \\
0.26 \\
0.25 \\
\end{array}$ & $\begin{array}{l}0.35 \\
0.33 \\
0.29 \\
0.38 \\
0.34 \\
0.33 \\
\end{array}$ & $\begin{array}{l}0.26 \\
0.26 \\
0.22 \\
0.24 \\
0.25 \\
0.23 \\
\end{array}$ & $\begin{array}{l}3.0 \\
2.7 \\
3.1 \\
3.4 \\
3.0 \\
3.2 \\
\end{array}$ \\
\hline Mean & & 0.27 & 0.29 & 0.17 & 0.27 & 0.34 & 0.24 & 3.0 \\
\hline SD & & 0.0183 & 0.0416 & 0.0192 & 0.0210 & 0.0221 & 0.2418 & 0.1167 \\
\hline$t$ & & 0.7734 & 0.1306 & 1.9897 & 0.5307 & 0.7595 & 0.2533 & 0.9097 \\
\hline $\mathrm{P}$ & & 0.4572 & 0.8987 & 0.0778 & 0.6072 & 0.4594 & 0.8057 & 0.9097 \\
\hline
\end{tabular}

Table 2: In vivo results.

D1 = depth deviation at the level of the implant shoulder; D2 = depth deviation at the implant center; D3 = depth deviation at the level of apical point; SMR $=$ shoulder mesial radial deviation; $\mathrm{SDR}=$ shoulder distal radial deviation; $\mathrm{ARP}=$ apex radial deviation at the level of apical point; $\mathrm{AD}=\mathrm{Angular}$ deviation 
Citation: Guarnieri R, Ippoliti S, Stefanelli LV, Di Carlo S, Pompa G (2016) In Vitro and In Vivo Accuracy Evaluation of Computer Guide Surgery for Dental Implant Placement Associated with Three Mini-implants Used as Precision Pins. Int J Surg Surgical Porced 1: 112. doi: http://dx.doi.org/10.15344/ijssp/2016/112

Page 3 of 3

In vivo test: The mean angular deviation between the virtual and actual positions of twelve placed implants was 3.06 degrees (SD 1.61). The mean D1 was $0.27 \mathrm{~mm}$ (SD 0.18), the mean D2 deviation was 0.29 $\mathrm{mm}$ (SD 0.25), and the mean $\mathrm{D} 3$ deviation was $0.17 \mathrm{~mm}$ (SD 0.15). The mean ARP deviation was $0.19 \mathrm{~mm}$ (SD 0.34); while at the entry point the mean SRM and SDR deviations were $0.34 \mathrm{~mm}$ (SD 0.16), and $0.24 \mathrm{~mm}$ (SD 0.13), respectively. The differences were found not statistically significant. In table 2 are reported the in vivo results. The maximum SMR error, the maximum SDR error, and the maximum ARP error were $0.31 \mathrm{~mm}, 0.38 \mathrm{~mm}$, and $0.29 \mathrm{~mm}$, respectively. The maximum D1, D2; and D3 error was $0.30 \mathrm{~mm}, 0.32 \mathrm{~mm}$, and 0.20 $\mathrm{mm}$, respectively. The maximum angle error was 3.8 degrees.

\section{Discussion}

The literatures reporting accuracy of guided surgery have been recently reviewed and analyzed by some researchers $[3,12,13]$, with the aim to summarize the available data. Vas Assche et al. [13], updating the previously systematic reviews on accuracy of computer-aided implant placement, reports nineteen studies which met the inclusion criteria. Meta analysis revealed a mean error of $0.99 \mathrm{~mm}$ (maximum $6.5 \mathrm{~mm}$ ) at the entry point and of $1.24 \mathrm{~mm}$ (maximum $6.9 \mathrm{~mm}$ ) at the apex. The mean angular deviation was 3.81 degrees (maximum $24.9^{\circ}$ degrees). Because of different study designs (human versus cadaver or model, drill holes versus implants, different evaluation methods), it was not been possible to identify one system as superior or inferior to others. In general, the accuracy reported was better in studies with models and cadavers than in studies with humans. This can be explained by better access, better visual control of the axis of the osteotomy, no movement of the patient, and any saliva or blood in the preclinical models. Furthermore, there was no significant difference between cadavers and models; therefore, the influence of the material (bone versus acrylic) might be negligible for testing the accuracy in a preclinical model. To assess the accuracy of the implant systems the following parameters were selected [13]:

a) deviation error in a horizontal direction at the entry point of the drill or implant;

b) deviation error in a horizontal direction at the apex of the drill or implant;

c) deviation in height (vertical direction);

d) deviation of the axis of the drill or implant

In the present study, these parameters were evaluated by the same operator, using the same apparatus and setting. Because the same method of image acquisition was used preoperatively and postoperatively, any error in the methodology for making the surgical guide and overlapping images was minimized.

In accuracy evaluation of 3D guided implant systems, it is important to evaluate the maximum deviation, which in vivo applications is crucial to prevent damage of anatomical structures. In the in vitro part of the present study, the maximum apical point error was 0.18 $\mathrm{mm}$, while Sarment et al. [14] reported a maximum apical error of $1.6 \mathrm{~mm}$. The same parameter in a studies of Dreiseidler et al. [15] and Soares et al.[16] was of $0.62 \mathrm{~mm}$, and $0.71 \mathrm{~mm}$, respectively. All these studies presented more pronounced mean and maximum differences than were observed in the present study. The our observed variations in the linear and angular measurements seem to be the results of more accurate positioning of the registration and surgical guides obtained in the present study by means of mini-implants, used as fiducial markers. The reproducibility of the template position during radiographic data acquisition and during implantation is a delicate issue, especially in edentulous patients. Several studies reported significantly better accuracy in partially edentulous patients than in totally edentulous patients, probably because the instability of the surgical template [1618]. The findings of our study confirm the data previously published data reporting that the use of three mini-implants, previously placed as reference elements, might improve the accuracy of the implant positioning after placement by guided surgery [19].

However, it must be remembered that the accuracy of guided surgery is not only depend by precise and stable repositioning of the registration and surgical template, but also by the sum of other cumulative error $[11,20]$ occurring during the image acquisition and data processing [1], during the template $\mathrm{CAD} / \mathrm{CAM}$ production [17], and during the drilling phase, caused by the bur-cylindre gap [20], and by attrition of sleeves and drills, after longer use [18]. As it is crucial to understand the significance of each step, and to realize the magnitude of the cumulated inaccuracy, other studies are needed. The accuracy of the method demonstrated in the present in vitro and in vivo study is within acceptable limits for dental implant guided surgery described in the literature. The use of three mini implants as referent pins, allows obtaining an high grade of precision during the computer guided surgery. However, further research, comparing the technique used in the present study with other methods as control group, are needed to confirm our results.

\section{Conclusions}

Considering the limitations of the present study, results, the in vitro and in vivo investigated the computer guided implant surgery showed that the use of three mini-implants used as precision pins, allows obtaining a promising accuracy in dental implant placement.

\section{Competing Interests}

The authors declare that they have no competing interests.

\section{Funding}

This study was sponsored with a grant by BioHorizons, Birmingham, AL, USA.

\section{References}

1. Dula K, Mini R, van der Stelt PF, Buser D (2001) The radiographic assessment of implant patients: decision-making criteria. Int $\mathrm{J}$ Oral Maxillofac Implants 16: 80-89.

2. Besimo CE, Lambrecht JT, Guindy JS (2000) Accuracy of implant treatment planning utilizing template-guided reformatted computed tomography. Dentomaxillofac Radiol 29: 46-51.

3. Jung RE, Schneider D, Ganeles J, Wismeijer D, Zwahlen M, et al. (2009) Computer technology applications in surgical implant dentistry: a systematic review. Int J Oral Maxillofac Implants 24: 92-109.

4. Fortin T, Bosson JL, Isidori M, Blanchet E (2006) Effect of flapless surgery on pain experienced in implant placement using an image-guided system. Int J Oral Maxillofac Implants 21: 298-304.

5. Ruppin J, Popovic A, Strauss M, Spüntrup E, Steiner A, et al. (2008) Evaluation of the accuracy of three different computer-aided surgery systems in dental implantology: optical tracking vs. stereolithographic splint systems. Clin Oral Implants Res 19: 709-716.

6. Mozzo P, Procacci C, Tacconi A, Martini PT, Andreis IA (1998) A new volumetric ct machine for dental imaging based on the conebeam technique: preliminary results. Eur Radiol 8: 1558-1564. 
Citation: Guarnieri R, Ippoliti S, Stefanelli LV, Di Carlo S, Pompa G (2016) In Vitro and In Vivo Accuracy Evaluation of Computer Guide Surgery for Dental Implant Placement Associated with Three Mini-implants Used as Precision Pins. Int J Surg Surgical Porced 1: 112. doi: http://dx.doi.org/10.15344/ijssp/2016/112

Page 3 of 3

7. Ludlow JB, Ivanovic M (2008) Comparative dosimetry of dental CBCT devices and 64-slice CT for oral and maxillofacial radiology. Oral Surg Oral Med Oral Pathol Oral Radiol Endod 106: 106-114.

8. van Steenberghe D, Glauser R, Blombäck U, Andersson M, Schutyser F, et al. (2005) A computed tomographic scan-derived customized surgical template and fixed prosthesis for flapless surgery and immediate loading of implants in fully edentulous maxillae: a prospective multicenter study. Clin Implant Dent Relat Res 7 Suppl 1: S111-S120.

9. Sanna AM, Molly L, van Steenberghe D (2007) Immediately loaded cad-cam manufactured fixed complete dentures using flapless implant placement procedures: a cohort study of consecutive patients. J Prosthet Dent 97: 331-339.

10. Komiyama A, Klinge B, Hultin M (2008) Treatment outcome of immediately loaded implants installed in edentulous jaws following computer- assisted virtual treatment planning and flapless surgery. Clin Oral Implants Res 19: 677-685.

11. Widmann G, Bale RJ (2006) Accuracy in computer-aided implant surgery-a review. Int J Oral Maxillofac Implants 21: 305-313.

12. Schneider D, Marquardt $P$, Zwahlen M, Jung RE (2009) A systematic review on the accuracy and the clinical outcome of computer-guided templatebased implant dentistry. Clin Oral Impl Res 20: 73-86.

13. Van Assche N1, Vercruyssen M, Coucke W, Teughels W, Jacobs R, et al (2012) Accuracy of computer-aided implant placement. Clin Oral Implants Res 23 Suppl 6: 112-123.

14. Sarment DP1, Sukovic P, Clinthorne N (2003) Accuracy of implant placement with a stereolithographic surgical guide. Int $\mathrm{J}$ Oral Maxillofac Implants 18: 571-577.

15. Fitzgerald M, O'Sullivan M, O'Connell B, Houston F (2010) Accuracy of bone mapping and guided flapless implant placement in human cadavers using a model-based planning procedure. Int $\mathrm{J}$ Oral Maxillofac Implants 25: 999-1006.

16. van Steenberghe D, Naert I, Andersson M, Brajnovic I, Van Cleynenbreugel $\mathrm{J}$, et al. (2002) A custom template and definitive prosthesis allowing immediate implant loading in the maxilla: a clinical report. Int $\mathrm{J}$ Oral Maxillofac Implants 17: 663-670.

17. Dreiseidler T, Neugebauer J, Ritter L, Lingohr T, Rothamel D, et al. (2009) Accuracy of a newly developed integrated system for dental implant planning. Clin Oral Implants Res 20: 1191-1199.

18. Horwitz J, Zuabi O, Machtei EE (2009) Accuracy of a computerized tomography-guided template-assisted implant placement system: an in vitro study. Clin Oral Implants Res 20: 1156-1162.

19. Tahmaseb A, De Clerck R, Wismeijer D (2009) Computer-guided implan placement: 3D planning software, fixed intraoral reference points, and CAD/ CAM technology. A case report. Int J Oral Maxillofac Implants 24: 541-546.

20. Widmann G, Stoffner R, Bale R (2009) Errors and error management in image-guided craniomaxillofacial surgery. Oral Surg Oral Med Oral Pathol Oral Radiol Endod 107: 701-715.

21. Valente F, Schiroli G, Sbrenna A (2009) Accuracy of computer-aided ora implant surgery: a clinical and radiographic study. Int J Oral Maxillofac Implants 24: 234-242. 